\title{
Gold nanoparticles: A Novel Application of Spectral Imaging in Proteomics - Preliminary Results
}

\author{
Heidelinde R. C. Dietrich ${ }^{a}$, Ian T. Young ${ }^{a}$, and Yuval Garini ${ }^{a}$ \\ ${ }^{a}$ Department of Imaging Science and Technology, Faculty of Applied Sciences, Delft University of \\ Technology, Lorentzweg 1, 2628 CJ Delft, The Netherlands
}

\begin{abstract}
The intense research in proteomics is demanding for fast, reliable and easy-to-use methods in order to study the proteome. In this proceeding we report the development of such a novel research tool based on spectral imaging and Resonance Light Scattering gold particles. This method will allow the study of DNA-protein interactions. We suggest a broad range of applications: the screening of proteins binding to a specific DNA sequence, the analysis of binding affinities between protein and DNA, and the investigation of the influence of environmental conditions on the binding. We will explain the principle, first experiments and first results based on Brownian motion.
\end{abstract}

Keywords: Gold Nanoparticles, Single Molecule Detection, Surface Plasmons, Proteomics, DNA-Protein Interactions, Protein-Protein Interactions

\section{INTRODUCTION}

In 2003 the Human Genome Project (HGP) was finally announced to be finished. The goal of this project amongst others was to determine the sequence of the human DNA (3 billion base pairs) and the identification of all human genes (app. 25,000). Despite the huge amount of data it is obvious that this was only the beginning of the study of the way that the cell functions at the molecular level. Wasinger et al. ${ }^{1}$ were the first to define the proteome as the "total protein complement for a genome". In general, the proteome is described as the total amount of proteins expressed by the genetic material of an organism in a particular cell type under specific environmental conditions.

Proteomics $^{2,3}$ is the study of proteins on the large scale typically by means of biochemical methods. Before the postgenomic era proteomics took advantage of two-dimensional polyacrylamide gels in order to analyze cell lysates and to visualize differential protein expressions. With time new techniques evolved such as mass spectrometry and protein chips. With the post-genomic period the definition of proteomics was broadened and differentiated. Proteomics includes large-scale identification and localization studies of proteins and the studies of interactions. Structural proteomics however only focuses on the study of protein structure. Today, the molecular research ${ }^{4}$ on proteins expressed in a healthy or diseased cell or tissue cannot be imagined without proteomics. That's due to the fact that many diseases not necessarily originate from a defect in a gene but from abnormal protein expression and localization. This specially applies for cancer diagnosis. Certain proteins can be used in order to detect different types of cancer, e.g. breast cancer or prostate cancer.

DNA-protein interactions ${ }^{5,6}$ are of high interest due to their importance in the control of cellular processes. These interactions include transcription regulation, DNA replication and repair etc. The study of the interaction of proteins with DNA (double or single stranded) can be divided into several aspects like: screening of proteins binding to a specific DNA sequence, identification of a DNA recognition sequence, analysis of binding affinities between protein and DNA, investigation of the influence of environmental conditions on the binding and investigation of DNA binding events controlling specific tasks like gene expression. There are a number of different methods for the analysis of DNA-protein interactions such as DNA footprinting ${ }^{5,6}$, electrophoretic mobility shift assays (EMSA), DNA-protein photocrosslinking, surface plasmon resonance (SPR). All of these methods are extensively used and each of them has its advantage and 
disadvantage. SPR ${ }^{7-10}$ provides a neat technique to study DNA-protein interactions especially the kinetics and affinity of binding events. SPR is a real-time, non destructive monitoring platform with no need for molecular labeling.

The intense search in proteomics demands for new analytical techniques that are reliable, fast, and easy to use. Spectral imaging and resonance light scattering gold nanoparticles seemed to be ideal candidates to tackle this issue. In this proceeding we consider the application of spectral imaging in proteomics. We will discuss first results and the issues of setting up such a method.

\section{THE PRINCIPLES}

Spectral imaging not only provides a two-dimensional image but also the spectrum at each point (or pixel) of a selected area. We use spectral imaging in combination with gold nanoparticles. These particles have the property to scatter light at different wavelengths depending on their size. We use $78 \mathrm{~nm}$ large gold beads that scatter yellow light. The idea behind this method lies in the characteristic of a metal nanoparticle. A metal nanoparticle scatters light due to the plasmon-photon interaction. The plasmon is due to the collaborative and coherent oscillation of electrons on the metalbead surface (Mie scattering) $)^{11}$. As soon as the particle approaches a metal surface, such as gold, electrons in the metal bead and metal surface will interact with each other. This will result ${ }^{12}$ in changes in the scattering frequency (wavelength) as well as changes in the intensity (Figure 1,2).

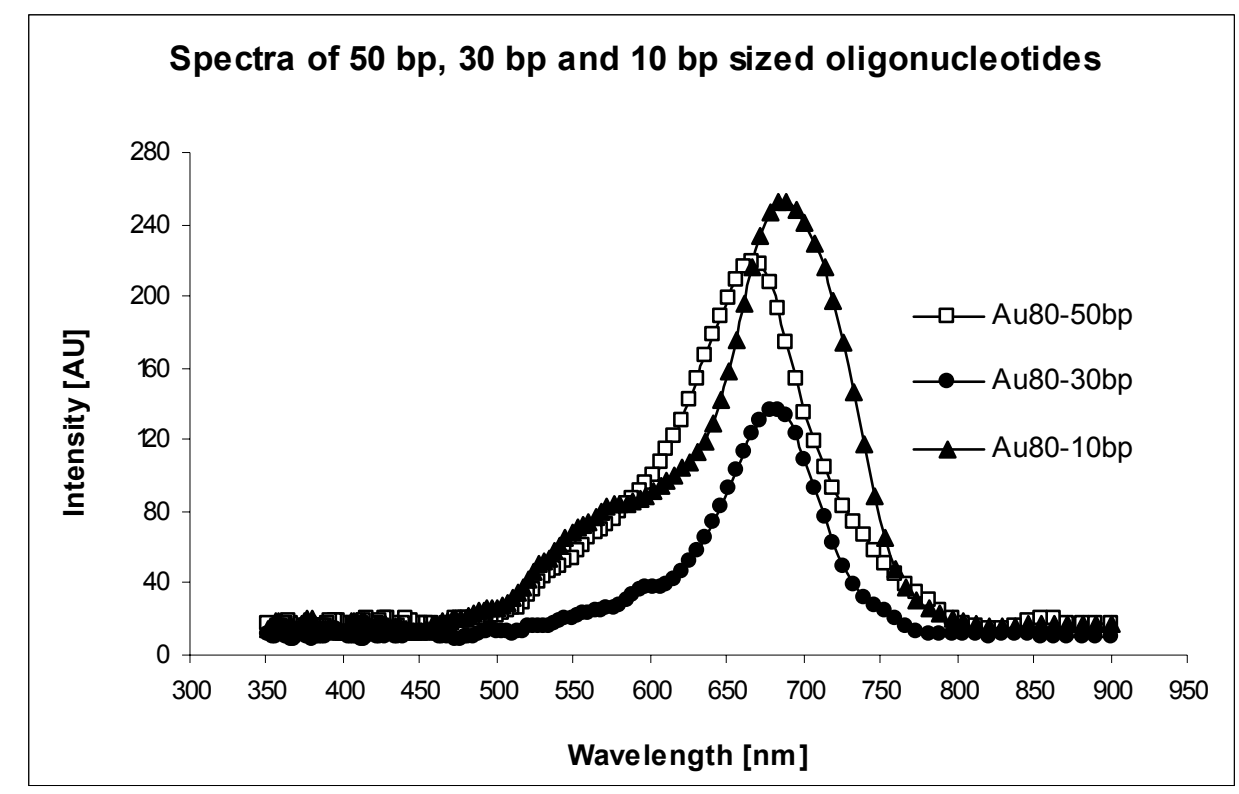

Figure 1: Spectra of three different sized oligonucleotides measured with a SpectraCube ${ }^{\circledR}$.

Some theoretical work has already been performed to calculate the wavelength shift as a function of the bead-surface separation. Based on this feature, it should be possible to establish a calibration curve of spatial position versus wavelength for different applications, such as the characterization of DNA-protein interactions. 


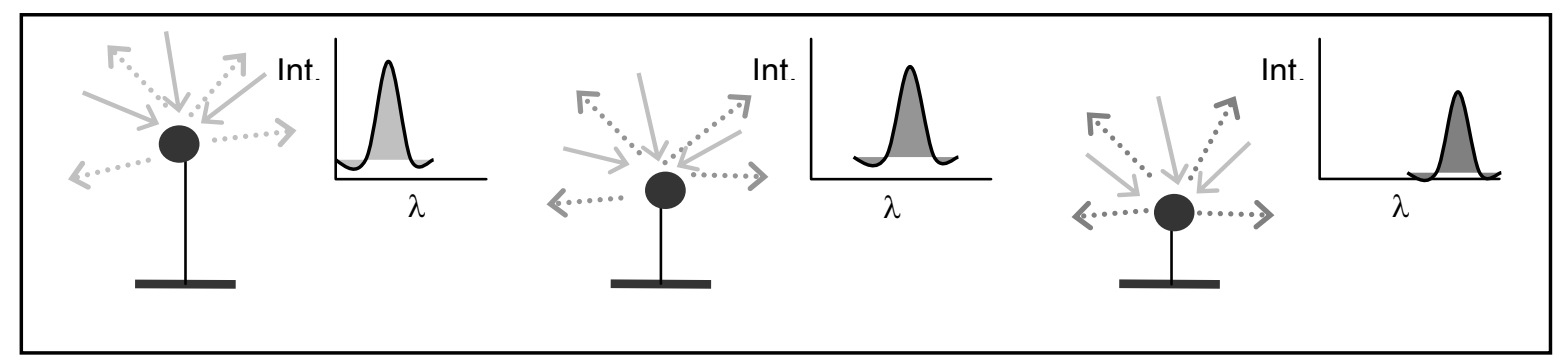

Figure 2: The intensity and wavelength of the scattered light changes as a function of the length of DNA.

When the calibration exist, it is then possible to follow the dynamic of the bead that is bound to DNA (or other protein). By recording the scattered intensity from the bead, it is possible to find the center of it and therefore get the $\mathrm{x}, \mathrm{y}$ position of the bead. Even though the observed size on a CCD will have the typical size of the point spread function (PSF), it is still possible to allocate the center of it with high precision, higher than the possible optical resolution of the optical system being used (this is not to be confused with optical resolution; here we are observing a single bead only, and therefore it is possible to achieve high precision in it's position). The intensity itself provides information on the $\mathrm{z}$ position. Together, it is therefore possible to follow the dynamics of the bead in three dimensions.

\section{SET-UP}

In order to detect the scattered light of the gold particles it is necessary to use darkfield illumination. At this moment, we are using a non-optimal darkfield illumination set-up (Figure 3). A fiber waveguide is used as the only illumination source. The white light illuminates the gold chip by an angle of approximately $45^{\circ}$. The scattered light is monitored by a 12 bit CCD camera (Hamamatsu, Orca-ER). The CCD array consists of 1344 x 1024 pixels. Each pixel has a size of 6.45 $\mathrm{x} 6.45 \mathrm{\mu m}^{2}$. The whole monitoring platform is based on a Leica microscope (DM RXA2) whereas the fluorescent filters are usually removed from the light path. A $20 \mathrm{x} / 0.5$ objective is used to collect the scattered light.

For spectral measurements and analysis, a spectral imaging system is used, SpectraCube ${ }^{\circledR}$ from Applied Spectral Imaging (ASI) (Figure 4). The spectral images are analyzed by SpectralView ${ }^{\mathrm{TM}}$ also from ASI. The integration time depends on the brightness of each measurement.

When following the DNA and bead dynamics, gray-level images are measured with a CCD. On an average 500 frames are taken for one series of images at an integration time of 100 milliseconds. The CCD camera is controlled by SimplePCI from Compix Inc. Imaging Systems. The collected images are analyzed by MATLAB.

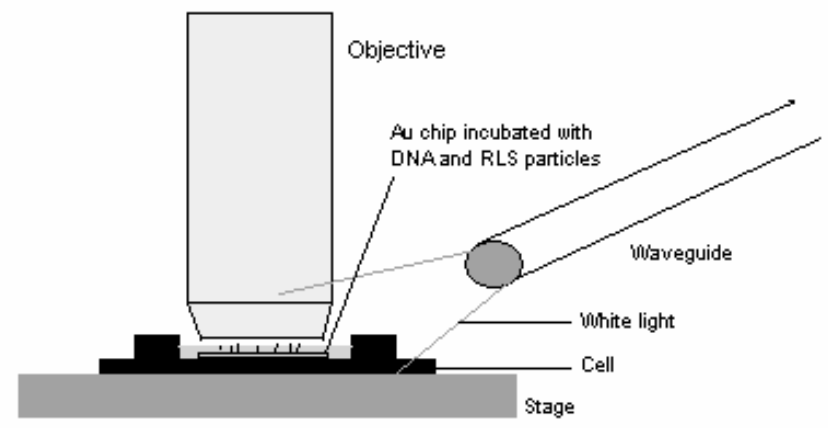

Figure 3: Scheme of the darkfield illumination setup. Only the stage area is shown. A fiber waveguide is used as the only illumination source. The white light illuminates the gold chip by an angle of approximately $45^{\circ}$. The scattered light is monitored by a 12 bit CCD camera. 

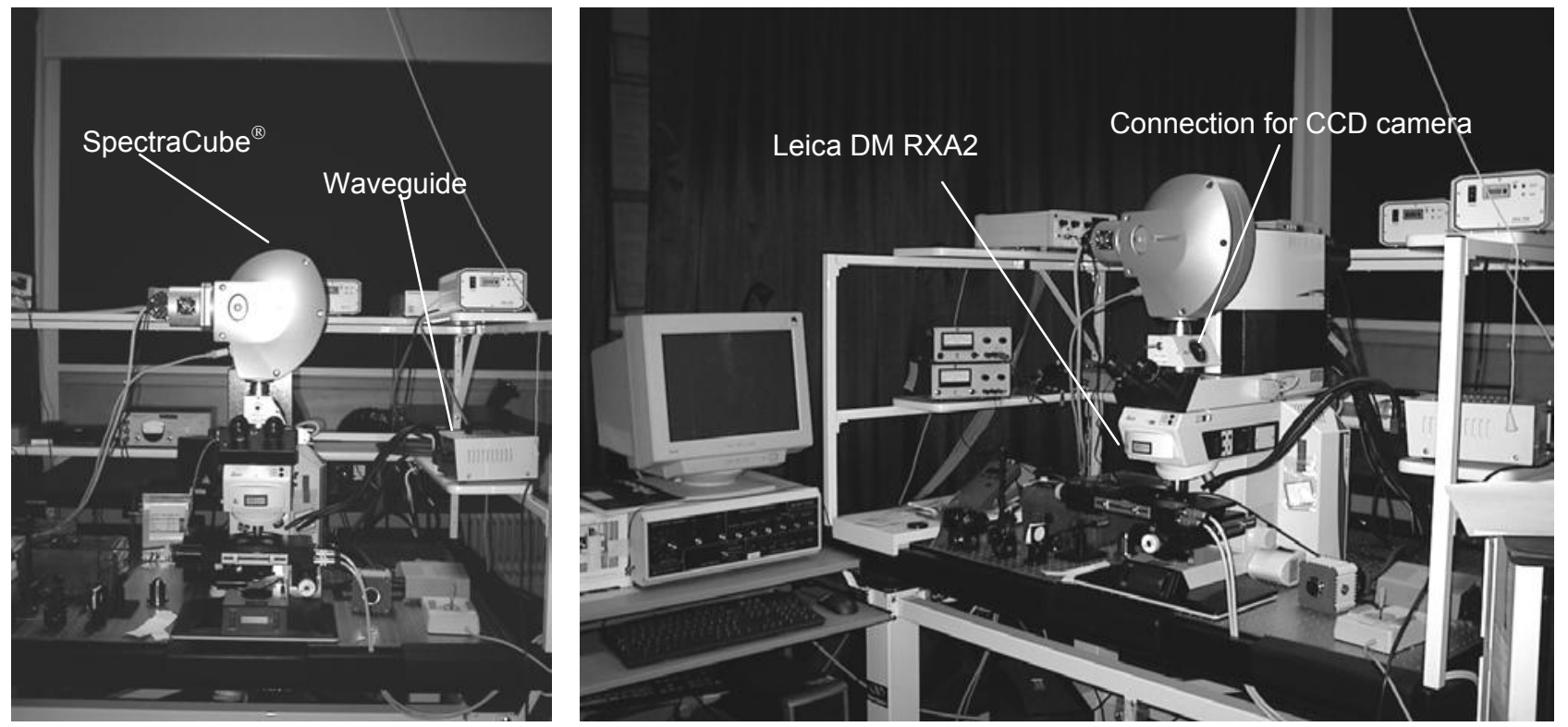

Figure 4: Monitoring platform. SpectraCube ${ }^{\circledR}$ mounted on a Leica DM RXA2. It allows to measure the spectrum at each pixel of the image.

\section{FIRST EXPERIME'NTS}

First experiments are based on the detection and analysis of the Brownian motion of dsDNA labeled with Resonance Light Scattering ${ }^{\mathrm{TM}}$ (RLS) gold particles (Invitrogen, The Netherlands). These experiments were meant to detect different lengths of double strand DNA. The differences in length should influence the motion pattern of the bead and the intensity of the scattered light. The freedom of movement of a free DNA strand without the interaction of a protein is much higher than that of a DNA that interacted with a protein. As a matter of fact, this allows the study of the interaction of proteins with single or double stranded DNA, the screening of proteins binding to a specific DNA sequence, analysis of binding affinities between protein and DNA, and the investigation of the influence of environmental conditions on the binding itself.
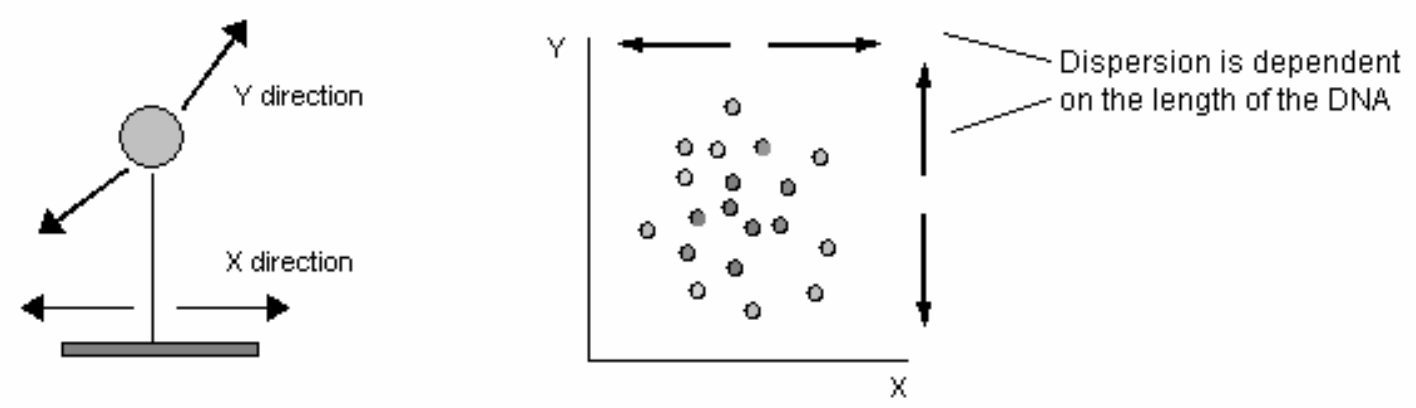

Figure 5: Motion pattern of the RLS particle as a function of the length of DNA.

Two different lengths of dsDNA were used: $5000 \mathrm{bp}$ and $1500 \mathrm{bp}$. These fragments were amplified from a lambda DNA template using the Expand Long Template PCR System (Roche). Both fragments have a 5-end thiol and a 5-end biotin modification. The anti-biotin labeled RLS particles bind to the free biotin on the dsDNA. The thiol end binds covalently to a gold surface (thiol-gold strength: $40 \mathrm{kcal} / \mathrm{mole}$ ). 


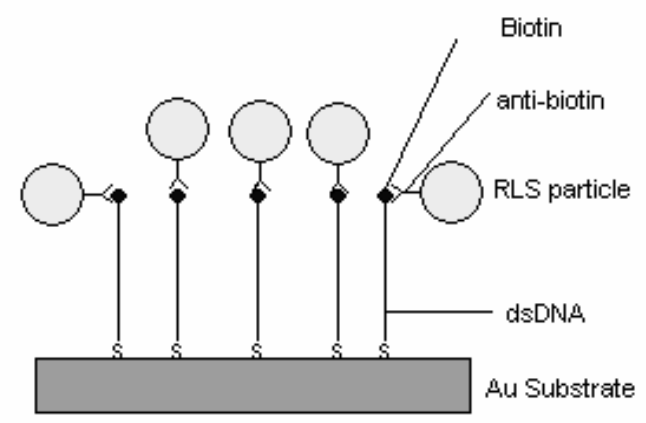

Figure 6: Double strand DNA labeled with RLS particles and immobilized on a gold surface.

All gold substrates are made of cleaved mica with a $150 \mathrm{~nm}$ thick gold layer (Molecular Imaging, distributed by ScienTec, France). These Au chips are flame annealed prior use and show a 111 surface structure.

Two gold chips were incubated with each DNA fragment and the gold particles and placed into a self-made chip holder. The precise adjustments of the set-up were already explained above in section 3 .

\section{FIRST RESULTS}

Both chips were scanned at several regions in order to obtain an overall view of the two surfaces thus, the influence of the length of the DNA on the dispersion of the gold particles and the intensity of the scattered light. The movement of the dsDNA labeled with RLS particles could be easily detected by the CCD and was even visible through the microscopes eyepieces.

Gold has a positive valence and attracts the negatively charged backbone of the dsDNA. This is a well-known effect. It is possible to diminish this effect by the application of a spacer molecule, which acts as a passivating agent for the gold surface. In most cases 6-mercapto-1-hexanol $(\mathrm{MCH})$ is used ${ }^{13-16}$. However, it can happen that the passivating layer does not cover the gold surface completely and that some DNA strands still get attached to the gold surface. In this case, the labeled RLS beads do not move anymore but still produce a very bright spot. Also the gold surface itself can generate spots that look like the ones produced from the gold particles. Nevertheless, the signal derived from gold particles labeled on a free-standing dsDNA can be clearly distinguished from "false-positive" spots.

A greater problem seems to be dust or dirt, which can alternatively bind to the DNA instead of the particles. It is much more difficult to distinguish between a real signal and one derived from dust or dirt particles. However, after close examination we can conclude that labeled RLS particles appear much smaller than dust or dirt. Figure 6 shows the Brownian motion of such a DNA labeled dirt particle. The length of the dsDNA was $1.5 \mu \mathrm{m}$. 


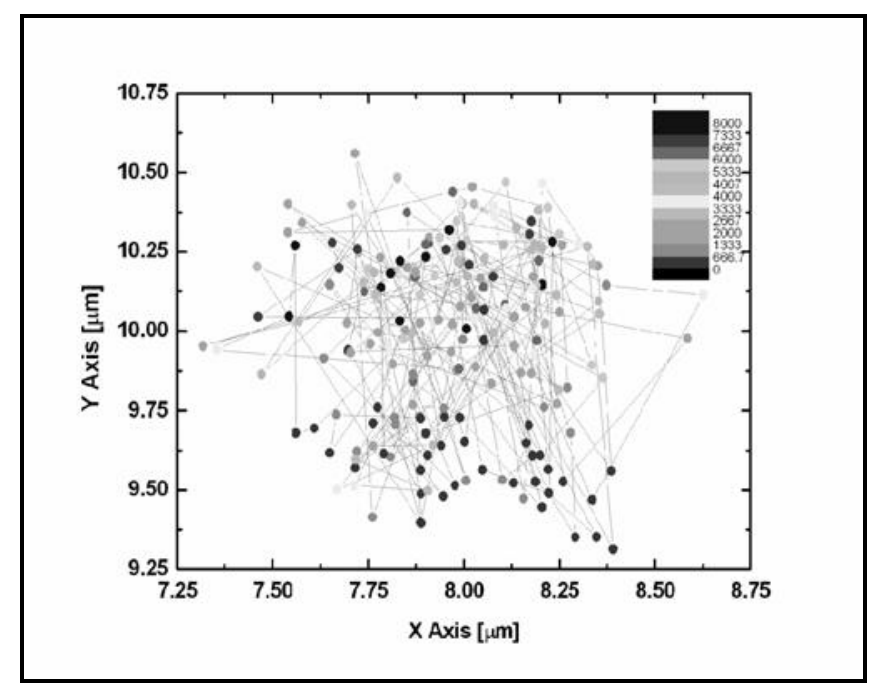

Figure 7: Brownian motion of a dirt particle bound to a $1.5 \mu \mathrm{m}$ long dsDNA. The different grey values represent the scattered light intensity.

The maximum movement in the $\mathrm{X}$ direction is $1320 \mathrm{~nm}$ and on the $\mathrm{Y}$ direction $1350 \mathrm{~nm}$. A free-standing DNA strand plus gold bead should give a theoretical distance of approximately $3000 \mathrm{~nm}$. From this follows that the negatively charged backbone of the DNA strand was most likely partially attached to the gold surface or that the dirt particle is heavier than the gold bead and pushes the DNA downwards to the surface.

On the same chip were also RLS particles monitored. As mentioned before the maximum travel distance of the bead on a $1.5 \mu \mathrm{m}$ long DNA should be 3000nm. In this experiment all analyzed particles had a dynamic range of about $25 \mathrm{~nm}$ in the $\mathrm{X}$ axis and $15 \mathrm{~nm}$ along the $\mathrm{Y}$ axis (Figure 8a). These numbers are comparable to spots on the surface that either derive from unmovable gold particles or from imperfections of the gold substrate (Figure 8b). This result gives a clear hint of the dimensions of a particle that is not free and hence not moving. However, it is striking that RLS particles immobilized on the gold surface blink. Such an immobilization could be due to a total attachment of the DNA strand on the gold chip.
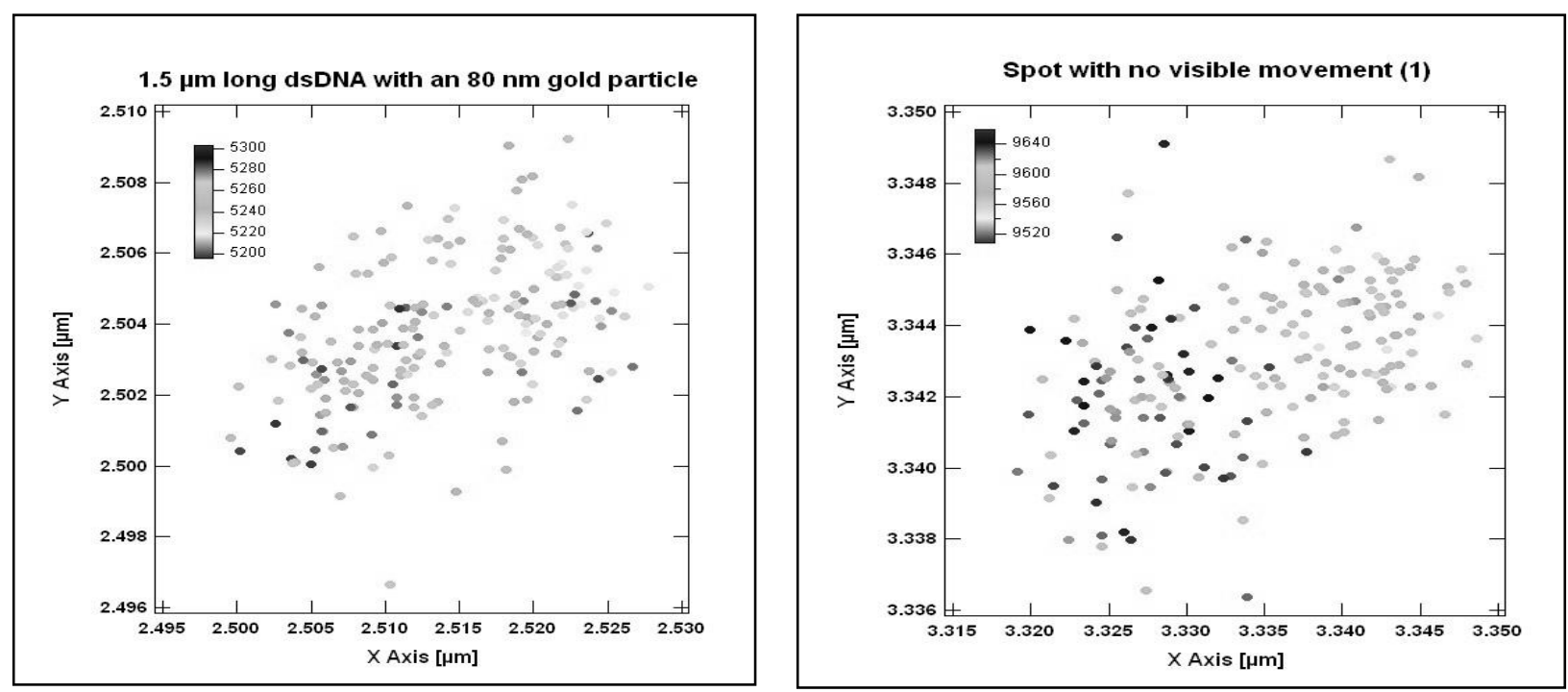

Figure 8: (a) Movement of a RLS particle on a $1.5 \mu \mathrm{m}$ long DNA strand. (b) Spot on the surface with no movement at all and no blinking. 
A RLS particle labeled on a $450 \mathrm{~nm}$ long dsDNA should have the freedom to move approximately $900 \mathrm{~nm}$ in both directions. This behavior was monitored. Two different beads on the same chip were analyzed and show comparable values. For both particles hold a movement maximum in the $\mathrm{X}$ direction of $800 \mathrm{~nm}$ and $1300 \mathrm{~nm}$ and in the $\mathrm{Y}$ direction approximately $1000 \mathrm{~nm}$.
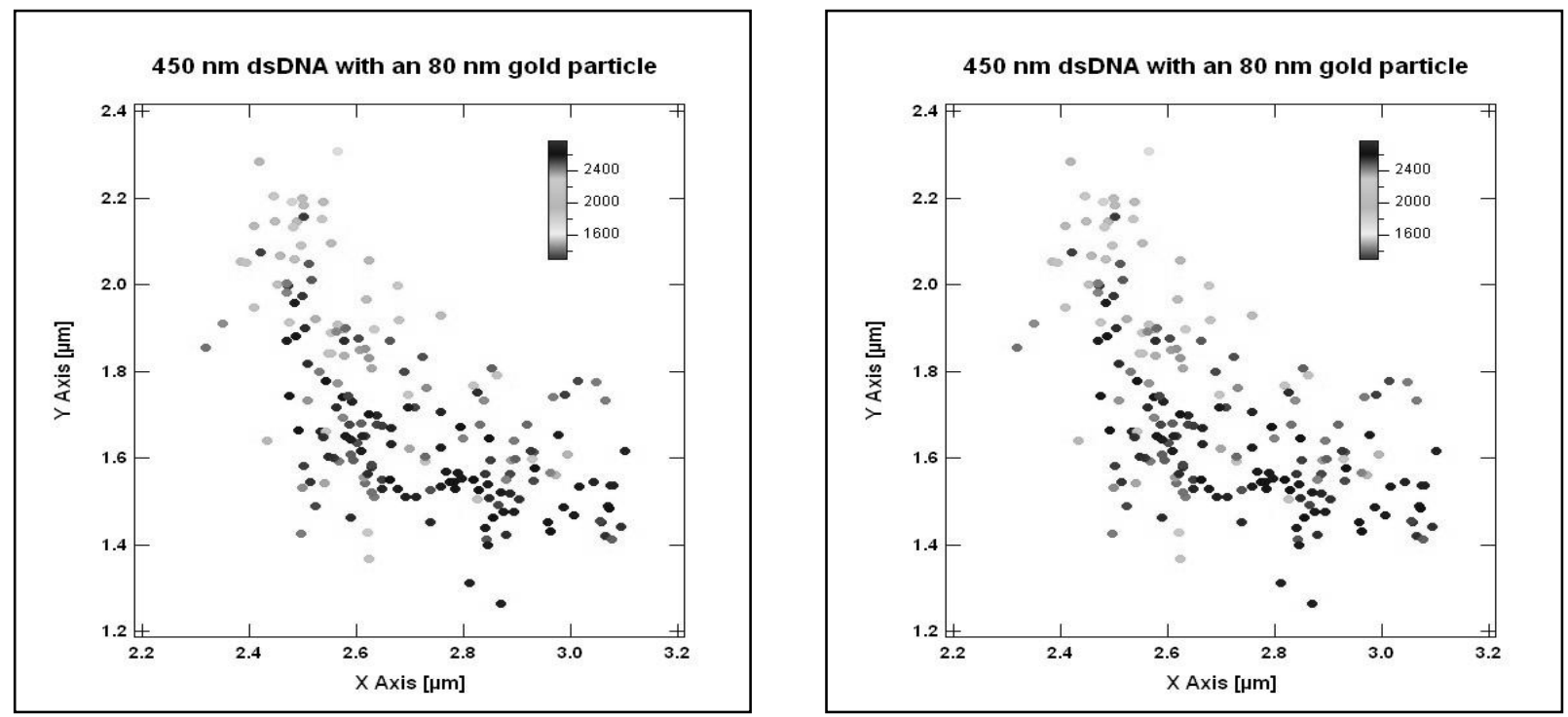

Figure 9: Brownian motion of a $450 \mathrm{~nm}$ long dsDNA fragment bound to an $80 \mathrm{~nm}$ gold particle.

\section{SUMMARY AND CONCLUSIONS}

We are able to monitor $80 \mathrm{~nm}$ large RLS particles immobilized on dsDNA with darkfield illumination. Sports derived from gold particle are clearly distinguishable from ones that are immobilized on the gold surface and from imperfections of the gold surface itself. In short, RLS particles that do not point into the substrate, thus particles that are not free, blink. However, visible spots derived from other sources that gold beads do not blink and have a dynamic movement of about $30 \mathrm{~nm}$.

Due to the results from the $1.5 \mu \mathrm{m}$ long DNA it can be concluded that our method has an error of $\pm 10 \mathrm{~nm}$ in $\mathrm{X}$ and $\mathrm{Y}$ directions. The experiment with $450 \mathrm{~nm}$ long DNA gave the expected dynamic behavior of about $1000 \mathrm{~nm}$.

We will further develop the detection platform and we are going to apply the system on DNA-binding proteins such as recA.

\section{ACKNOWLEDGEMENTS}

This work was partially supported by the Delft Inter-Faculty Research Center LifeTech and by the MicroNet program of NWO.

\section{REFERENCES}

1. V. C. Wasinger, S. J. Cordwell, A. Cerpa-Poljak, J.X. Yan, A. A. Gooley, M. R. Wilkins, M. W. Duncan, R. Harris, K. L. Williams, and I. Humphery-Smith, "Progress with gene-product mapping of the Mollicutes: Mycoplasma genitalium", Electrophoresis 16:7, pp. 1090-1094, 1995. 
2. A. Pandey, and M. Mann, "Proteomics to study genes and genomes", Nature 405, pp. 837-846, 2000.

3. B. Domon, and S. Broder, "Implications of New Proteomics Strategies for Biology and Medicine", J. Proteome. Res. 3:2, pp. 253-260, 2004.

4. I. A. Brewis, "Proteomics in reproductive research", Hum. Reprod. 14:3, pp. 2927-2929, 1999.

5. M. J. Guille, and G. G. Kneale, "Methods for the Analysis of DNA-Protein Interactions", Mol. Biotechnol. 8, pp. 3552, 1997.

6. J. S. Shumaker-Parry, C. T. Campell, G. D. Stormo, F. S. Silbaq, and R. H. Aebersold, "Probing Protein:Interactions Using a Uniform Monolayer of DNA and Surface Plasmon Resonance", Scanning and Force Microscopies for Biomedical Applications II, E. Tamiya and E. S. Yeung ed., Proceedings of SPIE 3922, pp. 1605-7422, 2000.

7. B. Persson, K. Stenhag, P. Nilsson, A. Larsson, M. Uhlén, and P. Nygren, "Analysis of Oligonucleotide Probe Affinities Using Surface Plasmon Resonance: A Means for Mutational Scanning”, Anal. Biochem. 246, pp. 34-44, 1997.

8. C. Bamdad, "A DNA Self-Assembled Monolayer for the Specific Attachment of Unmodified Double- or SingleStranded DNA", Biophys. J. 75, pp. 1997-2003, 1998.

9. M. Buckle, R. M. Williams, M. Negroni, and H. Buc, "Real time measurements of elongation by a reverse transcriptase using surface plasmon resonance", Proc. Nat. Acad. Sci. USA 93, pp. 889-894, 1996.

10. P. Y. Tsoi, and M. Yang, "Surface plasmon resonance study of the molecular recognition between polymerase and DNA containing various mismatches and conformational changes of DNA-protein complexes", Biosens. Bioelectron. 19, pp. 1209-1218, 2004.

11. J. Yguerabide and E. E. Yguerabide, "Light-Scattering Submicroscopic Particles as Highly Fluorescent Analogs and Their Use as Tracer Labels in Clinical and Biological Applications". Anal. Biochem. 262, pp. 137-156, 1998.

12. P. Nordlander and E. Prodan, "Plasmon hybridization in nanoparticles near metallic surfaces", Nano Lett. 4, pp. 2209-2213, 2004.

13. A. B. Steel, T. M. Herne, and M. J. Tarlov, "Electrochemical Quantitation of DNA Immobilized on Gold", Anal. Chem. 70, pp. 4670-4677, 1998.

14. A. B. Steel, R. L. Levicky, T. M. Herne, and M. J. Tarlov, "Immobilization of Nucleic Acids at Solid Surfaces: Effect of Oligonucleotide Length on Layer Assembly", Biophys. J. 79, pp. 975-981, 2000.

15. S. Moses et al., "Characterization of Single- and Double-Stranded DNA on Gold Surfaces", Langmuir 20, pp. 1113440, 2004.

16. R. Levicky, T. J. Herne, M. J. Tarlov, and S. K. Satija, "Using Self-Assembly To Control the Structure of DNA Monolayers on Gold: A Neutron Reflectivity Study”, J. Am. Chem. Soc. 120, pp. 9787-9792, 1998. 